\title{
Sign-changing solution for a third-order boundary-value problem in ordered Banach space with lattice structure
}

\section{Xiuli Lin* and Zengqin Zhao}

${ }^{*}$ Correspondence:

lin-xiuli78@163.com

School of Mathematical Sciences,

Qufu Normal University, Qufu,

273165, P.R. China

\begin{abstract}
In this paper, the sign-changing solution of a third-order two-point boundary-value problem is considered. By calculating the eigenvalues and the algebraic multiplicity of the linear problem and using a new fixed point theorem in an ordered Banach space with lattice structure, we give some conditions to guarantee the existence for a sign-changing solution.
\end{abstract}

Keywords: third-order boundary-value problem; sign-changing solution; Green's function; fixed point; lattice

\section{Introduction}

In this paper, we consider the following nonlinear third-order two-point boundary-value problem

$$
\left\{\begin{array}{l}
-u^{\prime \prime \prime}(t)=f(t, u(t)), \quad t \in[0,1] \\
u(0)=u^{\prime}(0)=u^{\prime}(1)=0
\end{array}\right.
$$

where $f \in C([0,1] \times R, R)$.

The study on the existence of the sign-changing solutions for the boundary-value problem is very useful and interesting both in theory and in application. Recently, there has been much attention focused on the problem, especially to the two-point or multi-point boundary-value problem. For the second-order two-point or multi-point boundary-value problem, many beautiful results have been given on the existence and multiplicity of the sign-changing solutions (see [1-5] and the references therein). For example, Xu and Sun [1] obtained an existence result of the sign-changing solutions for the second-order threepoint boundary-value problem

$$
\left\{\begin{array}{l}
-u^{\prime \prime}(t)=f(u), \quad t \in[0,1], \\
u(0)=0, \quad \alpha u(\eta)=u(1),
\end{array}\right.
$$

where $0<\alpha<1,0<\eta<1, f \in C(R, R)$. Xu [2] considered the sign-changing solutions for the second-order multi-point boundary-value problem

$$
\left\{\begin{array}{l}
-u^{\prime \prime}(t)=f(u), \quad t \in[0,1], \\
u(0)=0, \quad u(1)=\sum_{i=1}^{m-2} \alpha_{i} u\left(\eta_{i}\right),
\end{array}\right.
$$

O2014 Lin and Zhao; licensee Springer. This is an Open Access article distributed under the terms of the Creative Commons Attribution License (http://creativecommons.org/licenses/by/2.0), which permits unrestricted use, distribution, and reproduction in any medium, provided the original work is properly cited. 
where $\alpha_{i}>0, i=1,2, \ldots, m-2,0<\eta_{1}<\cdots<\eta_{m-2}<1, f \in C(R, R)$. In [4], Zhang and Sun obtained the existence and multiplicity of the sign-changing solutions for the integral boundary-value problem

$$
\left\{\begin{array}{l}
-u^{\prime \prime}(t)=f(u), \quad t \in[0,1], \\
u(0)=0, \quad u(1)=\int_{0}^{1} a(s) u(s) d s,
\end{array}\right.
$$

where $f \in C(R, R), a \in L(0,1)$ is nonnegative with $\int_{0}^{1} a^{2}(s) d s<1$. For the integral boundaryvalue problem (1.2), Li and Liu [5] also obtained the existence and multiplicity of the signchanging solutions in ordered Banach space with the lattice structure.

For the third-order boundary-value problem, the existence and multiplicity of solutions have also been discussed in many papers (see [6-11] and the references therein). However, the research on the sign-changing solutions has been proceeded slowly. For the problem (1.1), Yao and Feng $[10,11]$ established several existence results for the solutions including the positive solutions using the lower and upper solutions and a maximum principle, respectively. To our knowledge, however, there are fewer papers considered the signchanging solutions of the problem (1.1). Motivated by the work mentioned above, using the eigenvalues of linear operator, we give an existence result for the sign-changing solutions of the problem (1.1).

The main contribution of this paper are as follows: (a) for the sign-changing solutions of the problem (1.1), to our knowledge, there is no result using the eigenvalues of the linear operator until now; (b) we obtain the eigenvalues and the algebraic multiplicity of the linear problem corresponding the problem (1.1), which is one of the key points that we can use to prove our main result; (c) some conditions are given to guarantee the existence for a sign-changing solution of the problem (1.1).

\section{Notations and preliminaries}

The following results will be used throughout the paper.

Let $E=C[0,1],\|u\|=\max _{t \in[0,1]}|u(t)|$. Then $E$ is a real Banach space with the norm $\|\cdot\|$. Let $P=\{u \in E: u(t) \geq 0, t \in[0,1]\}$, and $P$ is a normal solid cone of $E,\{u \in E: u(t)>0, t \in$ $(0,1]\} \subset \stackrel{\circ}{P}=\{x \in P \mid x$ is an interior point of $P\}$.

Let the operators $K, F, A$ be defined by

$$
(K u)(t)=\int_{0}^{1} G(t, s) u(s) d s, \quad(F u)(t)=f(t, u(t)), \quad \text { for } t \in[0,1], u \in E,
$$

and $A=K F$, respectively, where

$$
G(t, s)=\frac{1}{2} \begin{cases}2 t s-s^{2}-t^{2} s, & 0 \leq s \leq t \leq 1 \\ t^{2}(1-s), & 0 \leq t \leq s \leq 1\end{cases}
$$

Remark 1 (1) $A, K: E \rightarrow E$ are completely continuous. (2) $G(t, s) \geq 0, t, s \in[0,1]$. In fact, since it is obvious in the other case, we only need to prove the case $0 \leq s \leq t \leq 1$. Now we suppose that $0 \leq s \leq t \leq 1$. Then

$$
G(t, s)=\frac{1}{2}\left(2 t s-s^{2}-t^{2} s\right)=\frac{1}{2} s\left(\left(t-t^{2}\right)+(t-s)\right) \geq 0 .
$$


Definition 2.1 [12] We call $E$ a lattice under the partial ordering $\leq$, if $\sup \{x, y\}$ and $\inf \{x, y\}$ exist for arbitrary $x, y \in E$.

Remark $2 E=C[0,1]$ is a lattice under the partial ordering $\leq$ that is deduced by the cone $P=\{u \in E: u(t) \geq 0, t \in[0,1]\}$ of $E$.

Definition 2.2 [12] Let $E$ be a Banach space with a cone $P, A: E \longrightarrow E$ be a nonlinear operator. We call that $A$ is a unilaterally asymptotically linear operator along $P_{w}=\{x \in E$ : $x \geq w, w \in E\}$, if there exists a bounded linear operator $L$ such that

$$
\lim _{x \in P_{w},\|x\| \rightarrow \infty} \frac{\|A x-L x\|}{\|x\|}=0 .
$$

$L$ is said to be the derived operator of $A$ along $P_{w}$ and will be denoted by $A_{P_{w}}^{\prime}$. Similarly, we can also define a unilaterally asymptotically linear operator along $P^{w}=\{x \in E: x \leq w, w \in$ $E\}$. Specially, if $w=\theta$, We call that $A$ is a unilaterally asymptotically linear operator along $P$ and $-P$.

Definition 2.3 [12] Let $D \subseteq E$ and $A: D \longrightarrow E$ be a nonlinear operator. $A$ is said to be quasi-additive on lattice, if there exists $v^{*} \in E$ such that

$$
A x=A x_{+}+A x_{-}+v^{*}, \quad \forall x \in D,
$$

where $x_{+}=x^{+}=\sup \{x, \theta\}, x_{-}=x^{-}=-\sup \{-x, \theta\}$.

Remark 3 It is easy to see that the operators $F$ and $A=K F$ defined by (2.1) are both quasiadditive on the lattice $E=C[0,1]$.

Let us list some conditions and preliminary lemmas to be used in this paper.

$\left(\mathrm{H}_{1}\right) f \in C([0,1] \times R, R)$ is strictly increasing in $u$, and $f(t, u) u>0$ for all $t \in[0,1], u \in$ $R \backslash\{0\}$.

$\left(\mathrm{H}_{2}\right) \lim _{|u| \rightarrow+\infty} \frac{f(t, u)}{u}=\beta_{1}$ uniformly on $[0,1]$. There exists a positive integer $n_{1}$ such that

$$
\lambda_{2 n_{1}}^{3}<\beta_{1}<\lambda_{2 n_{1}+1}^{3}
$$

where $0<\lambda_{1}<\lambda_{2}<\cdots<\lambda_{n}<\cdots$ are the positive solutions of the equation

$$
-\frac{1}{2} e^{-\frac{3}{2} \lambda}=\cos \left(\frac{\sqrt{3}}{2} \lambda+\frac{2 \pi}{3}\right)
$$

$\left(\mathrm{H}_{3}\right) \lim _{u \rightarrow 0} \frac{f(t, u)}{u}=\beta_{0}$ uniformly on $[0,1]$, and $0<\beta_{0}<\lambda_{1}^{3}$.

Lemma 2.1 For any $f \in C[0,1], u \in C^{3}[0,1]$ is a solution of the following problem:

$$
\left\{\begin{array}{l}
-u^{\prime \prime \prime}(t)=f(t), \quad t \in[0,1] \\
u(0)=u^{\prime}(0)=u^{\prime}(1)=0
\end{array}\right.
$$


if and only if $u(t)$ is a solution of the integral equation

$$
u(t)=\int_{0}^{1} G(t, s) f(s) d s
$$

where $G(t, s)$ is defined by (2.2).

Proof On the one hand, integrating the equation

$$
-u^{\prime \prime \prime}(t)=f(t), \quad t \in[0,1]
$$

over $[0, t]$ for three times, we have

$$
u(t)=-\frac{1}{2} \int_{0}^{t}(t-s)^{2} f(s) d s+\frac{1}{2} u^{\prime \prime}(0) t^{2}+u^{\prime}(0) t+u(0) .
$$

Then

$$
u^{\prime}(t)=-t \int_{0}^{t} f(s) d s+\int_{0}^{t} s f(s) d s+u^{\prime \prime}(0) t+u^{\prime}(0) .
$$

Combining them with boundary condition $u(0)=u^{\prime}(0)=u^{\prime}(1)=0$, we conclude that

$$
u^{\prime \prime}(0)=\int_{0}^{1}(1-s) f(s) d s
$$

Therefore,

$$
\begin{aligned}
u(t) & =-\frac{1}{2} \int_{0}^{t}(t-s)^{2} f(s) d s+\frac{1}{2} t^{2} \int_{0}^{1}(1-s) f(s) d s \\
& =-\frac{1}{2} \int_{0}^{t}\left[(t-s)^{2}-t^{2}(1-s)\right] f(s) d s+\frac{1}{2} t^{2} \int_{t}^{1}(1-s) f(s) d s \\
& =\int_{0}^{1} G(t, s) f(s) d s .
\end{aligned}
$$

On the other hand, since

$$
u(t)=\int_{0}^{1} G(t, s) f(s) d s=\frac{1}{2} \int_{0}^{t}\left(2 t s-s^{2}-t^{2} s\right) f(s) d s+\frac{1}{2} \int_{t}^{1} t^{2}(1-s) f(s) d s,
$$

therefore,

$$
\begin{aligned}
& u^{\prime}(t)=\int_{0}^{t}(s-t s) f(s) d s+\int_{t}^{1} t(1-s) f(s) d s, \\
& u^{\prime \prime}(t)=-\int_{0}^{t} s f(s) d s+\int_{t}^{1}(1-s) f(s) d s,
\end{aligned}
$$

and

$$
u^{\prime \prime \prime}(t)=-t f(t)-(1-t) f(t)=-f(t)
$$

Moreover, we get $u(0)=u^{\prime}(0)=u^{\prime}(1)=0$. 
Remark 4 Considering Lemma 2.1, we find that $u$ is a solution of the problem (1.1) if and only if $u$ is a fixed point of the operator $A=K F$.

From the following lemma, we can obtain the eigenvalues and the algebraic multiplicity of the linear operator $K$.

Lemma 2.2 The eigenvalues of the linear operator $K$ are

$$
\frac{1}{\lambda_{1}^{3}}>\frac{1}{\lambda_{2}^{3}}>\cdots>\frac{1}{\lambda_{n}^{3}}>\cdots
$$

and the algebraic multiplicity of each positive eigenvalue $\frac{1}{\lambda_{n}^{3}}$ is equal to 1 , where $0<\lambda_{1}<$ $\lambda_{2}<\cdots<\lambda_{n}<\cdots$ are the positive solutions of (2.3).

Proof Let $\bar{\lambda}$ be a positive eigenvalue of the linear operator $K$, and $y \in E \backslash\{\theta\}$ be an eigenfunction corresponding to eigenvalue $\bar{\lambda}$. By Lemma 2.1, we have

$$
\left\{\begin{array}{l}
-y^{\prime \prime \prime}(t)=\frac{1}{\lambda} y(t), \quad t \in(0,1) \\
y(0)=y^{\prime}(0)=y^{\prime}(1)=0
\end{array}\right.
$$

The auxiliary equation of the differential equation (2.4) has roots $-\mu, \frac{1}{2} \mu(1+\sqrt{3} i), \frac{1}{2} \mu(1-$ $\sqrt{3} i$ ), where $\mu=\frac{1}{\sqrt[3]{\lambda}}$. Thus the general solution of (2.4) is

$$
y(t)=C_{1} e^{-\mu t}+C_{2} e^{\frac{1}{2} \mu t} \cos \left(\frac{\sqrt{3}}{2} \mu t\right)+C_{3} e^{\frac{1}{2} \mu t} \sin \left(\frac{\sqrt{3}}{2} \mu t\right) .
$$

Then

$$
y^{\prime}(t)=C_{1}\left(-\mu e^{-\mu t}\right)+C_{2} \mu e^{\frac{1}{2} \mu t} \cos \left(\frac{\sqrt{3}}{2} \mu t+\frac{\pi}{3}\right)+C_{3} \mu e^{\frac{1}{2} \mu t} \sin \left(\frac{\sqrt{3}}{2} \mu t+\frac{\pi}{3}\right)
$$

Applying the condition $y(0)=y^{\prime}(0)=0$, we obtain $C_{2}=-C_{1}, C_{3}=\sqrt{3} C_{1}$, where $C_{1} \neq 0$.

Applying the second condition $y^{\prime}(1)=0$, that is,

$$
e^{-\mu}+2 e^{\frac{1}{2} \mu} \cos \left(\frac{\sqrt{3}}{2} \mu+\frac{2 \pi}{3}\right)=0
$$

Considering (2.3), we see that $\mu$ is one of $\lambda_{1}, \lambda_{2}, \ldots, \lambda_{n}, \ldots$, that is,

$$
\frac{1}{\lambda_{1}^{3}}>\frac{1}{\lambda_{2}^{3}}>\cdots>\frac{1}{\lambda_{n}^{3}}>\cdots
$$

are eigenvalues of the linear operator $K$ and the eigenfunction corresponding to the eigenvalue $\frac{1}{\lambda_{n}^{3}}$ is

$$
y_{n}(t)=C\left(e^{-\lambda_{n} t}-e^{\frac{1}{2} \lambda_{n} t} \cos \left(\frac{\sqrt{3}}{2} \lambda_{n} t\right)+\sqrt{3} e^{\frac{1}{2} \lambda_{n} t} \sin \left(\frac{\sqrt{3}}{2} \lambda_{n} t\right)\right),
$$

where $C$ is a nonzero constant. 
Next we prove that the algebraic multiplicity of the eigenvalue $\frac{1}{\lambda_{n}^{3}}$ is 1 . From (2.6), any two eigenfunctions corresponding to the same eigenvalue $\frac{1}{\lambda_{n}^{3}}$ are merely nonzero constant multiples of each other, that is,

$$
\operatorname{dim} \operatorname{ker}\left(\frac{1}{\lambda_{n}^{3}} I-K\right)=\operatorname{dim} \operatorname{ker}\left(I-\lambda_{n}^{3} K\right)=1
$$

Now we show that

$$
\operatorname{ker}\left(I-\lambda_{n}^{3} K\right)=\operatorname{ker}\left(I-\lambda_{n}^{3} K\right)^{2} .
$$

Obviously, we only need to show that

$$
\operatorname{ker}\left(I-\lambda_{n}^{3} K\right)^{2} \subset \operatorname{ker}\left(I-\lambda_{n}^{3} K\right)
$$

In fact, for any $y \in \operatorname{ker}\left(I-\lambda_{n}^{3} K\right)^{2},\left(I-\lambda_{n}^{3} K\right) y$ is an eigenfunction of linear operator $K$ corresponding to the eigenvalue $\frac{1}{\lambda_{n}^{3}}$ if $\left(I-\lambda_{n}^{3} K\right) y \neq \theta$. Considering (2.6), there exists a nonzero constant $\sigma$ such that

$$
\left(I-\lambda_{n}^{3} K\right) y(t)=\sigma\left(e^{-\lambda_{n} t}-e^{\frac{1}{2} \lambda_{n} t} \cos \left(\frac{\sqrt{3}}{2} \lambda_{n} t\right)+\sqrt{3} e^{\frac{1}{2} \lambda_{n} t} \sin \left(\frac{\sqrt{3}}{2} \lambda_{n} t\right)\right) .
$$

By direct computation, we have

$$
\left\{\begin{array}{l}
y^{\prime \prime \prime}(t)+\lambda_{n}^{3} y(t) \\
\quad=-\sigma \lambda_{n}^{3}\left(e^{-\lambda_{n} t}-e^{\frac{1}{2} \lambda_{n} t} \cos \left(\frac{\sqrt{3}}{2} \lambda_{n} t\right)+\sqrt{3} e^{\frac{1}{2} \lambda_{n} t} \sin \left(\frac{\sqrt{3}}{2} \lambda_{n} t\right)\right), \quad t \in[0,1], \\
y(0)=y^{\prime}(0)=y^{\prime}(1)=0 .
\end{array}\right.
$$

It is easy to see that the solution for the corresponding homogeneous equation of (2.7) is of the form

$$
y_{0}(t)=C_{1} e^{-\lambda_{n} t}+C_{2} e^{\frac{1}{2} \lambda_{n} t} \cos \left(\frac{\sqrt{3}}{2} \lambda_{n} t\right)+C_{3} e^{\frac{1}{2} \lambda_{n} t} \sin \left(\frac{\sqrt{3}}{2} \lambda_{n} t\right)
$$

Then, by an ordinary differential equation method, we see that the general solution of (2.7) is of the form

$$
y(t)=y_{0}(t)+y_{1}^{*}(t)+y_{2}^{*}(t), \quad t \in[0,1]
$$

where

$$
y_{1}^{*}(t)=-\frac{1}{3} \sigma \lambda_{n} t e^{-\lambda_{n} t}
$$

is the special solution of the equation

$$
y^{\prime \prime \prime}(t)+\lambda_{n}^{3} y(t)=-\sigma \lambda_{n}^{3} e^{-\lambda_{n} t},
$$


and

$$
\begin{aligned}
y_{2}^{*}(t) & =\frac{1}{3} \sigma \lambda_{n} t e^{\frac{1}{2} \lambda_{n} t}\left(\cos \left(\frac{\sqrt{3}}{2} \lambda_{n} t\right)+\sqrt{3} \sin \left(\frac{\sqrt{3}}{2} \lambda_{n} t\right)\right) \\
& =\frac{2}{3} \sigma \lambda_{n} t e^{\frac{1}{2} \lambda_{n} t} \cos \left(\frac{\pi}{3}-\frac{\sqrt{3}}{2} \lambda_{n} t\right)
\end{aligned}
$$

is the special solution of the equation

$$
y^{\prime \prime \prime}(t)+\lambda_{n}^{3} y(t)=\sigma \lambda_{n}^{3} e^{\frac{1}{2} \lambda_{n} t}\left(\cos \left(\frac{\sqrt{3}}{2} \lambda_{n} t\right)-\sqrt{3} \sin \left(\frac{\sqrt{3}}{2} \lambda_{n} t\right)\right) .
$$

Then

$$
\begin{aligned}
\left(y_{1}^{*}\right)^{\prime}(t)= & -\frac{1}{3} \sigma \lambda_{n} e^{-\lambda_{n} t}+\frac{1}{3} \sigma \lambda_{n}^{2} t e^{-\lambda_{n} t}, \\
\left(y_{2}^{*}\right)^{\prime}(t)= & \frac{2}{3} \sigma \lambda_{n} e^{\frac{1}{2} \lambda_{n} t} \cos \left(\frac{\pi}{3}-\frac{\sqrt{3}}{2} \lambda_{n} t\right)+\frac{1}{3} \sigma \lambda_{n}^{2} t e^{\frac{1}{2} \lambda_{n} t} \cos \left(\frac{\pi}{3}-\frac{\sqrt{3}}{2} \lambda_{n} t\right) \\
& +\frac{\sqrt{3}}{3} \sigma \lambda_{n}^{2} t e^{\frac{1}{2} \lambda_{n} t} \sin \left(\frac{\pi}{3}-\frac{\sqrt{3}}{2} \lambda_{n} t\right) .
\end{aligned}
$$

Applying the condition $y(0)=y^{\prime}(0)=0$, we obtain $C_{2}=-C_{1}, C_{3}=\sqrt{3} C_{1}$. From the condition $y^{\prime}(1)=0$, we obtain

$$
\left(y_{0}\right)^{\prime}(1)+\left(y_{1}^{*}\right)^{\prime}(1)+\left(y_{2}^{*}\right)^{\prime}(1)=0 .
$$

From (2.5), we have $\left(y_{0}\right)^{\prime}(1)=0$. Thus it follows from (2.8) that

$$
\begin{aligned}
& -e^{-\lambda_{n}}+\lambda_{n} e^{-\lambda_{n}}+2 e^{\frac{1}{2} \lambda_{n}} \cos \left(\frac{\pi}{3}-\frac{\sqrt{3}}{2} \lambda_{n}\right)+\lambda_{n} e^{\frac{1}{2} \lambda_{n}} \cos \left(\frac{\pi}{3}-\frac{\sqrt{3}}{2} \lambda_{n}\right) \\
& +\sqrt{3} \lambda_{n} e^{\frac{1}{2} \lambda_{n}} \sin \left(\frac{\pi}{3}-\frac{\sqrt{3}}{2} \lambda_{n}\right)=0,
\end{aligned}
$$

which implies that

$$
e^{-\lambda_{n}}+e^{\frac{1}{2} \lambda_{n}} \cos \left(\frac{\pi}{3}-\frac{\sqrt{3}}{2} \lambda_{n}\right)+\sqrt{3} e^{\frac{1}{2} \lambda_{n}} \sin \left(\frac{\pi}{3}-\frac{\sqrt{3}}{2} \lambda_{n}\right)=0 .
$$

That is

$$
-\frac{1}{2} e^{-\frac{3}{2} \lambda_{n}}=\cos \frac{\sqrt{3}}{2} \lambda_{n}
$$

which is a contradiction of

$$
-\frac{1}{2} e^{-\frac{3}{2} \lambda_{n}}=\cos \left(\frac{\sqrt{3}}{2} \lambda_{n}+\frac{2 \pi}{3}\right)
$$

Therefore, the algebraic multiplicity of the eigenvalue $\frac{1}{\lambda_{n}^{3}}$ is 1 . 
Lemma 2.3 Suppose that $\left(\mathrm{H}_{1}\right)$ holds and $y \in P \backslash\{\theta\}$ is a solution of the (1.1), then $y \in \stackrel{\circ}{P}$. Similarly, if $y \in(-P) \backslash\{\theta\}$ is a solution of the (1.1), then $y \in-\stackrel{\circ}{P}$.

Proof The proof is obvious.

Lemma 2.4 Suppose that $\left(\mathrm{H}_{1}\right)-\left(\mathrm{H}_{3}\right)$ hold. Then the operator $A$ is Fréchet differentiable at $\theta$ and $\infty$, and $A_{\theta}^{\prime}=\beta_{0} K, A_{\infty}^{\prime}=\beta_{1} K$.

Proof Since $\left(\mathrm{H}_{3}\right): \lim _{u \rightarrow 0} \frac{f(t, u)}{u}=\beta_{0}$ uniformly on $[0,1]$. That is, for any $\varepsilon>0$, there exists $\delta>0$ such that

$$
\left|f(t, u)-\beta_{0} u\right| \leq \varepsilon|u|, \quad \forall t \in[0,1], 0<|u|<\delta .
$$

From $\left(\mathrm{H}_{1}\right)$, it is easy to see that $\forall t \in[0,1], f(t, 0)=0$. Then, for any $u \in E$ with $\|u\|<\delta$, we have

$$
\begin{aligned}
\left|\left(A u-A \theta-\beta_{0} K u\right)(t)\right| & =\left|K\left(F u-\beta_{0} u\right)(t)\right| \\
& \leq\|K\| \max _{s \in[0,1]}\left|f(s, u(s))-\beta_{0} u(s)\right| \leq\|K\|\|u\| \varepsilon
\end{aligned}
$$

Then

$$
\left\|A u-A \theta-\beta_{0} K u\right\| \leq\|K\|\|u\| \varepsilon, \quad\|u\|<\delta .
$$

Thus,

$$
\lim _{\|u\| \rightarrow 0} \frac{\left\|A u-A \theta-\beta_{0} K u\right\|}{\|u\|}=0,
$$

which means $A_{\theta}^{\prime}=\beta_{0} K$.

Since $\left(\mathrm{H}_{2}\right): \lim _{|u| \rightarrow+\infty} \frac{f(t, u)}{u}=\beta_{1}$ uniformly on $[0,1]$. That is, for any $\varepsilon>0$, there exists $R>0$ such that

$$
\left|f(t, u)-\beta_{1} u\right| \leq \varepsilon|u|, \quad \forall t \in[0,1],|u|>R .
$$

Let $M_{R}=\max _{0 \leq|u| \leq R}|f(t, u)|$. Then

$$
\left|(F u)(t)-\beta_{1} u(t)\right|=\left|f(t, u(t))-\beta_{1} u(t)\right| \leq M_{R}+\beta_{1} R+\varepsilon\|u\|, \quad t \in[0,1], u \in E .
$$

Thus,

$$
\left|\left(A u-\beta_{1} K u\right)(t)\right|=\left|K\left(F u-\beta_{1} u\right)(t)\right| \leq\|K\|\left(M_{R}+\beta_{1} R+\varepsilon\|u\|\right) .
$$

Then

$$
\lim _{\|u\| \rightarrow+\infty} \frac{\left\|A u-\beta_{1} K u\right\|}{\|u\|} \leq \varepsilon\|K\| .
$$

Therefore, $A_{\infty}^{\prime}=\beta_{1} K$. 
Remark 5 Suppose $\left(\mathrm{H}_{2}\right)$ holds. Similar to Lemma 2.4, we have

$$
A_{P}^{\prime}=A_{-P}^{\prime}=A_{\infty}^{\prime}=\beta_{1} K
$$

Lemma 2.5 [13] Suppose that $E$ is an ordered Banach space with a lattice structure, $P$ is a normal solid cone in $E$, and the nonlinear operator $A$ is quasi-additive on the lattice. Assume that

(i) $A$ is strongly increasing on $P$ and $-P$;

(ii) both $A_{P}^{\prime}$ and $A_{-P}^{\prime}$ exist with $r\left(A_{P}^{\prime}\right)>1$ and $r\left(A_{-P}^{\prime}\right)>1$; 1 is not an eigenvalue of $A_{P}^{\prime}$ or $A_{-P}^{\prime}$ corresponding a positive eigenvector;

(iii) $A \theta=\theta$; the Fréchet derivative $A_{\theta}^{\prime}$ of $A$ at $\theta$ is strongly positive, and $r\left(A_{\theta}^{\prime}\right)<1$;

(iv) the Fréchet derivative $A_{\infty}^{\prime}$ of $A$ at $\infty$ exists; 1 is not an eigenvalue of $A_{\infty}^{\prime}$; the sum $\beta$ of the algebraic multiplicities for all eigenvalues of $A_{\infty}^{\prime}$ lying in the interval $(1, \infty)$ is an even number.

Then $A$ has at least three nontrivial fixed points containing one sign-changing fixed point.

\section{Main result}

We state the main result of this paper.

Theorem 3.1 Suppose that $\left(\mathrm{H}_{1}\right)-\left(\mathrm{H}_{3}\right)$ hold. Then the problem (1.1) has at least three solutions including a sign-changing solution.

We need only to prove that $A=K F$ satisfies the four conditions of Lemma 2.5 .

Proof Noticing

$$
A_{\theta}^{\prime}=\beta_{0} K, \quad A_{P}^{\prime}=A_{-P}^{\prime}=A_{\infty}^{\prime}=\beta_{1} K .
$$

(i) $A$ is strongly increasing on $P$ and $-P$. In fact, from $\left(\mathrm{H}_{1}\right)$ and $K(P \backslash\{\theta\}) \subseteq \stackrel{\circ}{P}$, we see that $A$ is strongly increasing on $P$. Similarly, $A$ is strongly increasing on $-P$.

(ii) From $A_{P}^{\prime}=A_{-P}^{\prime}=\beta_{1} K, \lambda_{2 n_{1}}^{3}<\beta_{1}<\lambda_{2 n_{1}+1}^{3}$ and Lemma 2.2, we find that 1 is not an eigenvalue of $A_{P}^{\prime}$ or $A_{-P}^{\prime}$ and $r\left(A_{P}^{\prime}\right)=r\left(A_{-P}^{\prime}\right)=\frac{\beta_{1}}{\lambda_{1}^{3}}>1$.

(iii) From $A_{\theta}^{\prime}=\beta_{0} K, 0<\beta_{0}<\lambda_{1}^{3}, K(P \backslash\{\theta\}) \subseteq \stackrel{\circ}{P}$, Lemma 2.2 and $\left(\mathrm{H}_{1}\right)$, we have $A_{\theta}^{\prime}$ is strongly positive, $A \theta=\theta$ and $r\left(A_{\theta}^{\prime}\right)=\frac{\beta_{0}}{\lambda_{1}^{3}}<1$.

(iv) Since $A_{\infty}^{\prime}=\beta_{1} K, \lambda_{2 n_{1}}^{3}<\beta_{1}<\lambda_{2 n_{1}+1}^{3}$ and Lemma 2.2, the condition (iv) of Lemma 2.5 is satisfied.

Therefore, from Lemma 2.5, we see that $A$ has at least three nontrivial fixed points including one sign-changing fixed point. Then, the problem (1.1) has at least three solutions, including one sign-changing solution.

Example 3.1 Consider the following third-order boundary-value problem

$$
\left\{\begin{array}{l}
-u^{\prime \prime \prime}(t)=f(t, u(t)), \quad t \in[0,1] \\
u(0)=u^{\prime}(0)=u^{\prime}(1)=0
\end{array}\right.
$$


where

$$
f(t, u)= \begin{cases}300(u-1)+(1+t) \sqrt{u}+3, & t \in[0,1], u \in(1,+\infty), \\ (1+t) u^{\frac{5}{3}}+3 u, & t \in[0,1], u \in[-1,1], \\ 300(u+1)+(1+t) \sqrt[3]{u}-3, & t \in[0,1], u \in(-\infty,-1) .\end{cases}
$$

By simple calculations, we have $\lambda_{1} \approx 3.0167, \lambda_{2} \approx 6.6506, \lambda_{3} \approx 10.2782, \beta_{0}=3, \beta_{1}=$ 300. Then it is easy to see that $f(t, u)$ satisfies the conditions $\left(\mathrm{H}_{1}\right)-\left(\mathrm{H}_{3}\right)$. Therefore, the boundary-value problem (3.1) has at least three solutions, including one sign-changing solution.

\section{Competing interests}

The authors declare that they have no competing interests.

\section{Authors' contributions}

The authors declare that the study was realized in collaboration with the same responsibility. All authors read and approved the final manuscript.

\section{Acknowledgements}

The authors are highly grateful for the referees' careful reading and comments on this paper. The research is supported by Program for Scientific research innovation team in Colleges and universities of Shandong Province, the Doctoral Program Foundation of Education Ministry of China (20133705110003), the Natural Science Foundation of Shandong Province of China (ZR2011AM008, ZR2012AM010, ZR2012AQ024).

Received: 11 December 2013 Accepted: 7 April 2014 Published: 22 May 2014

\section{References}

1. $\mathrm{Xu}, \mathrm{X}, \mathrm{Sun}, \mathrm{J}$ : On sign-changing solution for some three-point boundary value problems. Nonlinear Anal. 59, 491-505 (2004)

2. Xu, X: Multiple sign-changing solutions for some m-point boundary value problems. Electron. J. Differ. Equ. 89, 1-14 (2004)

3. Guo, L, Sun, J, Zhao, Y: Existence of positive solutions for nonlinear third-order three-point boundary value problems. Nonlinear Anal. 68, 3151-3158 (2008)

4. Zhang, X, Sun, J: On multiple sign-changing solutions for some second-order integral boundary value problems. Electron. J. Qual. Theory Differ. Equ. 44, 1-15 (2010)

5. Li, H, Liu, Y: On sign-changing solution for a second-order integral boundary value problems. Comput. Math. Appl. 62, 651-656 (2011)

6. Feng, X, Feng, H, Bai, D: Eigenvalue for a singular third-order three-point boundary value problem. Appl. Math. Comput. 219, 9783-9790 (2013)

7. Guo, L, Sun, J, Zhao, Y: Existence of positive solutions for nonlinear third-order three-point boundary value problems. Nonlinear Anal. 68, 3151-3158 (2008)

8. Du, Z, Ge, W, Lin, X: Existence of solutions for a class of third-order nonlinear boundary value problems. J. Math. Anal. Appl. 294, 104-112 (2004)

9. Sun, Y: Positive solutions of singular third-order three-point boundary value problem. J. Math. Anal. Appl. 306 589-603 (2005)

10. Yao, Q, Feng, Y: The existence of solution for a third-order two-point boundary value problem. Appl. Math. Lett. 15, 227-232 (2002)

11. Feng, Y, Liu, S: Solvability of a third-order two-point boundary value problem. Appl. Math. Lett. 18, 1034-1040 (2005)

12. Sun, J, Liu, X: Computation of topological degree in ordered Banach spaces with lattice structure and its applications to superlinear differential equations. J. Math. Anal. Appl. 348, 927-937 (2008)

13. Liu, X, Sun, J: Computation of topological degree of unilaterally asymptotically linear operators and its applications. Nonlinear Anal. 71, 96-106 (2009) 